TITLE:

\title{
A coupled $K d V$ equation is one case of the four-reduction of the KP hierarchy
}

$\operatorname{AUTHOR}(\mathrm{S}):$

SATSUMA, Junkichi; HIROTA, Ryogo

\section{CITATION:}

SATSUMA, Junkichi ... [et al]. A coupled KdV equation is one case of the four-reduction of the KP hierarchy. 数理解析研究所講究録 1982, 472: 16-24

ISSUE DATE:

1982-11

URL:

http://hdl.handle.net/2433/103261

RIGHT: 
A coupled $\mathrm{KdV}$ equation is one case of the four-reduction of the KP hierarchy

Junkichi SATSUMA (Miyazaki Medical College) 限喥顺吉

RYogo HIROTA (Hiroshima University) 広田良吾

It is shown that the coupled $\mathrm{KdV}$ equation introduced by the present authors is a special case of the four-reduced $\mathrm{KP}$ hierarchy which is included in the general theory of $\tau$ functions. From the fact it is also shown that the soliton solutions can be derived from those of the KP equation.

\section{Four-reduction of the KP hierarchy}

Recently sato et al. developed the general theory of $\tau$ functions. ${ }^{1,2)}$ Their theory starts with a system of linear equations for an eigenfunction $\psi(x, \lambda)$,

$$
\begin{aligned}
& L(\mathrm{x}, \partial) \psi(\mathrm{x}, \lambda)=\lambda \psi(\mathrm{x}, \lambda), \\
& \frac{\partial}{\partial \mathrm{x}_{\mathrm{n}}} \psi(\mathrm{x}, \lambda)=B_{\mathrm{n}}(\mathrm{x}, \partial) \psi(\mathrm{x}, \lambda), \quad \mathrm{n}=1,2, \cdots
\end{aligned}
$$

The pseudo-differential operator $L^{\prime}$ is defined by

$$
L(x, \partial)=\partial+u_{2}(x) \partial^{-1}+u_{3}(x) \partial^{-2}+u_{4}(x) \partial^{-3}+\cdots,
$$

where $u_{n}(x)$ are functions in infinitely many time variables $\mathrm{x}=\left(\mathrm{x}_{1}, \mathrm{x}_{2}, \mathrm{x}_{3}, \mathrm{x}_{4}, \cdots\right)$ and $\partial$ denotes $\partial / \partial \mathrm{x}_{1}$. The operators $B_{\mathrm{n}}$ are defined as the differential part of $L(x, \partial)^{n}$. For example,

$$
B_{1}=\partial,
$$




$$
\begin{aligned}
B_{2} & =\partial^{2}+2 u_{2}, \\
B_{3} & =\partial^{3}+3 u_{2} \partial+3 u_{3}+3 u_{2, x^{\prime}} \\
B_{4}= & \partial^{4}+4 u_{2} \partial^{2}+\left(4 u_{3}+6 u_{2, x}\right) \partial+4 u_{4}+6 u_{3, x} \\
& +4 u_{2, x x}+6 u_{2}^{2},
\end{aligned}
$$

where subscript $\mathrm{x}$ denotes partial differentiation.

From the compatibility condition of (1) and (2), we have a series of nonlinear partial differential equations,

$$
\frac{\partial L}{\partial \mathbf{x}_{\mathrm{n}}}=\left[B_{\mathrm{n}}, L\right] \equiv B_{\mathrm{n}} L-L B_{\mathrm{n}^{\prime}}
$$

or equivalently,

$$
\frac{\partial B}{\partial \mathrm{x}_{\mathrm{n}}}-\frac{\partial B_{\mathrm{n}}}{\partial \mathrm{x}_{\mathrm{m}}}=\left[B_{\mathrm{n}}, B_{\mathrm{m}}\right] \text {. }
$$

For $\mathrm{n}=2$ and $\mathrm{m}=3$, as a nontrivial simple case, (6) gives the Kadomtsev-Petviashvili (KP) equation,

$$
\frac{\partial}{\partial x_{1}}\left(\frac{\partial u_{2}}{\partial x_{3}}-\frac{1}{4} \frac{\partial^{3} u_{2}}{\partial x_{1}}-3 u_{2} \frac{\partial u_{2}}{\partial x_{1}}\right)-\frac{3}{4} \frac{\partial^{2} u_{2}}{\partial x_{2}^{2}}=0
$$

Hence the series of equations is called the KP hierarchy.

If we demand that $u_{n}(n=1,2,3, \cdots)$ do not depend on $x_{\ell}, x_{2 \ell}, x_{3 \ell}, \cdots$ for a positive integer $\ell$, the system of equations is called the $\ell$-reduction of the $\mathrm{KP}$ hierarchy. In this case, $\psi(x, \lambda)$ satisfies

$$
\frac{\partial}{\partial x_{\ell}} \psi(x, \lambda)=\lambda^{\ell} \psi(x, \lambda)
$$

or

$$
B_{\ell}(x, \partial) \psi(x, \lambda)=\lambda^{\ell} \psi(x, \lambda)
$$


Here we study a simple case of the four-reduction. Let us consider the following system of linear equations;

$$
\begin{aligned}
& \frac{\partial}{\partial y} \psi=B_{2} \psi, \\
& \frac{\partial}{\partial t} \psi=B_{3} \psi, \\
& B_{4} \psi=\lambda^{4} \psi,
\end{aligned}
$$

where we have changed the variables as $x_{1}=x, x_{2}=y$ and $\mathrm{x}_{3}=t$ for convenience. The compatibility condition of (10) and (11) gives the KP equation. From (11) and (12) we have

$$
\begin{aligned}
u_{2, t}= & u_{2, x x x}+3 u_{3, x x}+3 u_{4, x}+6 u_{2} u_{2, x},(13 a) \\
u_{3, t}= & -\frac{3}{4} u_{2, x x x x}-2 u_{3, x x x}-\frac{3}{2} u_{4, x x}-\frac{9}{2} u_{2} u_{2, x x} \\
& -\frac{9}{2} u_{2, x}{ }^{2}-3 u_{2} u_{3, x}-3 u_{2, x} u_{3},(13 b) \\
u_{4, t}= & \frac{3}{8} u_{2, x x x x x}+\frac{3}{4} u_{3, x x x x}+\frac{1}{4} u_{4, x x x}+\frac{3}{4} u_{2} u_{2, x x x} \\
& +\frac{27}{4} u_{2, x} u_{2, x x}-3 u_{2} u_{3, x x}+\frac{9}{2} u_{2, x} u_{3, x} \\
& +\frac{3}{2} u_{2, x x} u_{3}-6 u_{2} u_{4, x}-3 u_{3} u_{3, x}-9 u_{2}^{2} u_{2, x}
\end{aligned}
$$

Introducing dependent variables $u, \phi, \omega$ as

$$
\begin{aligned}
& u_{2}=u_{1} \\
& u_{3}=-\frac{1}{2} u_{x}+\phi_{x^{\prime}} \\
& u_{4}=\frac{1}{4} u_{x x}-\frac{1}{2} u^{2}-\phi_{x x}-\phi^{2}+\omega_{,}
\end{aligned}
$$

(13) may be rewritten as

$$
u_{t}=\frac{1}{4} u_{x x x}+3 u_{x}+3\left(-\phi^{2}+\omega\right)_{x}^{\prime}
$$




$$
\begin{aligned}
& \phi_{t}=-\frac{1}{2} \phi_{x x x}-3 u \phi_{x} \\
& \omega_{t}=-\frac{1}{2} \omega_{x x x}-3 u \omega_{x} .
\end{aligned}
$$

If we take $\omega=0$ in (15), it reduces to a coupled KdV equation,

$$
\begin{aligned}
& u_{t}=\frac{1}{4} u_{x x x}+3 u u_{x}-6 \phi \phi_{x^{\prime}} \\
& \phi_{t}=-\frac{1}{2} \phi_{x x x}-3 u \phi_{x^{\prime}}
\end{aligned}
$$

on which we have shown the existence of the three soliton solution and five nontrivial conserved quantities. ${ }^{3)}$ This equation describes an interaction of two long waves with different dispersion relations.

From the viewpoint of inverse scattering transform, the system of linear equations (11) and (12) might be enough to solve an initial value problem of the coupled $\mathrm{KdV}$ equation. For the purpose of obtaining its soliton solutions, however, the general theory of $\tau$ functions is very powerful, since the solutions are given as a specialization of the soliton solutions of the KP equation.

In order to investigate the relationship between solutions of the coupled KdV and the KP equations, we may employ the compatibility condition of (10) and (12). After substituting (14), we find from (10) and (12),

$$
\begin{aligned}
& u_{y}=2 \phi_{x x^{\prime}} \\
& \phi_{y}=-2 \phi^{2}+\omega^{2} \\
& \phi_{Y Y}+\phi_{x x x x}+8 u \phi_{x x}+4 u_{x} \phi_{x}=0 .
\end{aligned}
$$


It is easily shown that (17c) is compatible with (15), (17a) and (17b). Substituting (17a) and (17b) into (15a) yields the KP equation,

$$
u_{x t}-\frac{1}{4} u_{x x x x}-3\left(u u_{x}\right)_{x}-\frac{3}{4} u_{y y}=0
$$

Thus we can conclude that the coupled KdV equation is a special case of the four-reduction of the $\mathrm{KP}$ equation and its solutions may be derived from those of the KP equation.

2. Soliton solutions of the coupled KdV equation

Here we derive several soliton solutions of the coupled $\mathrm{KdV}$ equation. For the purpose, it is most convenient to rewrite the equation in the bilinear form.

Introducing the dependent variable transformation,

$$
\begin{aligned}
& u=(\log f)_{x x^{\prime}} \\
& \phi=g / f, \\
& \omega=h / f
\end{aligned}
$$

(15) may be reduced to their bilinear forms,

$$
\begin{aligned}
& \left(D_{x} D_{t}-\frac{1}{4} D_{x}^{4}\right) f \cdot f=6\left(-g^{2}+f h\right), \\
& \left(D_{t}+\frac{1}{2} D_{x}^{3}\right) f \cdot g=0, \\
& \left(D_{t}+\frac{1}{2} D_{x}^{3}\right) f \cdot h=0,
\end{aligned}
$$

where we have used the binary operators defined by

$$
\left.D_{i}^{n} D_{j}^{m} a \cdot b \equiv\left(\frac{\partial}{\partial x_{i}}-\frac{\partial}{\partial x_{i}^{!}}\right)^{n}\left(\frac{\partial}{\partial x_{j}}-\frac{\partial}{\partial x_{j}^{\prime}}\right)^{m} a(x) b\left(x^{\prime}\right)\right|_{x=x^{\prime}}
$$

If $h$ is taken to be zero, (20a) and (20b) becomes essentially 
equivalent with the bilinear form of the coupled KdV equation in Ref.3. The subsidiary conditions (17a) and (17b) are simply written as

$$
\begin{aligned}
& f_{y}=2 g, \\
& g_{y}=2 h .
\end{aligned}
$$

Substituting (22) into (20) yields

$$
\begin{aligned}
& \left(D_{x} D_{t}-\frac{1}{4} D_{x}^{4}-\frac{3}{4} D_{y}^{2}\right) f \cdot f=0, \\
& D_{y}\left(D_{t}+\frac{1}{2} D_{x}^{3}\right) f \cdot f=0 \\
& \frac{\partial}{\partial y}\left[D_{y}\left(D_{t}+\frac{1}{2} D_{x}^{3}\right) f \cdot f\right]=0,
\end{aligned}
$$

the first of which is the KP equation itself and the second is one of the KP hierarchy.

Let us consider the soliton solutions of the KP equation. The N-soliton solution is written as

$$
\begin{aligned}
f_{N}= & +\sum_{j=1}^{N} a_{j} e^{\xi_{j}}+\sum_{1 \leq i<j \leq N} c_{i j} a_{i} a_{j} e^{\xi_{i}+\xi_{j}}+ \\
& +\cdots+\left({ }_{1 \leq i<j \leq N} c_{i j}\right) \prod_{j=1}^{N} a_{j} e^{\xi_{1}+\xi_{2}+\cdots+\xi_{N}},
\end{aligned}
$$

where

$$
\xi_{j}=\left(p_{j}-q_{j}\right) x+\left(p_{j}^{2}-q_{j}^{2}\right) y+\left(p_{j}^{3}-q_{j}^{3}\right) t
$$

and

$$
c_{i j}=\frac{\left(p_{i}-p_{j}\right)\left(q_{i}-q_{j}\right)}{\left(p_{i}-q_{j}\right)\left(q_{i}-p_{j}\right)} .
$$

Although the N-soliton solution from the general theory contains infinitely many time variables, we include only the 
terms of $x, y$ and $t$ in (25) since we are interested in the solutions of two of the four-reduced KP hierarchy, i.e. (23a) and $(23 b)$. The four-reduction requires

$$
p_{j}^{4}=q_{j}^{4}
$$

in the solution.

There are three nontrivial cases in choosing the form of the phase factor $\xi_{j}$;

$1)$ for $p_{j}=-q_{j}, \quad \xi_{j}=P_{j} x^{\prime}+\frac{1}{4} p_{j}^{3} t \quad\left(p_{j} \equiv 2 p_{j}\right)$,

2) for $p_{j}=i q_{j}, \quad \xi_{j}=P_{j} x-i p_{j}^{2} y-\frac{1}{2} p_{j}^{3} t \quad\left(p_{j} \equiv(1+i) p_{j}\right)$

3) for $p_{j}=-i q_{j^{\prime}} \quad \xi_{j}=P_{j} x+i p_{j}^{2} y-\frac{1}{2} p_{j}^{3} t \quad\left(p_{j:} \equiv(1-i) p_{j}\right)$

Consider the one-soliton solution,

$$
f=1+a_{1} \exp \xi_{1}
$$

If we choose the case 1), (29) reduces to

$$
f=1+a_{1} \exp \left(P_{1} x+\frac{1}{4} P_{1}^{3} t\right)
$$

From the conditions (22), we have $g=h=0$, i.e. $\phi=\omega=0$, in this case. Hence this solution corresponds to the KdV. soliton of the system of equations (15), which is explicitly written as

$$
u=\frac{p^{2}}{4} \operatorname{sech}^{2} \frac{1}{2}\left(P_{1} x+\frac{1}{4} P_{1}^{3} t\right)
$$

for $a_{1}=1$. Secondly, if we choose the case 2 ) or 3 ), we obtain

$$
f=1+a_{1} \exp \eta_{1}
$$




$$
\begin{aligned}
& g=\frac{1}{2} i a_{1} P_{1}^{2} \exp \eta_{1}, \\
& h=-\frac{1}{4} a_{1} P_{1}^{4} \exp \eta_{1}
\end{aligned}
$$

where $\eta_{1}=P_{1} x \mp i P_{1}^{2} y-\frac{1}{2} P_{1}^{3} t$. By taking $y=0$, this solution gives a steady solution of (15). However, since we can not choose $h=0$ with $g \neq 0$, this does not give a solution of the coupled $\mathrm{KdV}$ equation (16).

Next we consider the two-soliton solution of the KP equation,

$$
f=1+a_{1} \exp \xi_{1}+a_{2} \exp \xi_{2}+a_{1} a_{2} c_{12} \exp \left(\xi_{1}+\xi_{2}\right) \text {. }
$$

Choosing $\xi_{1}$ and $\xi_{2}$ as (28a), we have

$$
\begin{aligned}
& f=1+a_{1} e^{n_{1}}+a_{2} e^{\eta_{2}}+\left(\frac{p_{1}-p_{2}}{p_{1}+p_{2}}\right)^{2} a_{1} a_{2} e^{n_{1}+n_{2}}, \\
& g=h=0, \\
& n_{j}=P_{j} x+\frac{1}{4} P_{j}^{3} t,
\end{aligned}
$$

which correspond to the KdV two-soliton solution. By choosing $\xi_{1}$ as (28b) and $\xi_{2}$ as $(28 c)$, and taking $P_{1}=P_{2}, a_{1}=-a_{2}=\sqrt{2} i$, (33) reduces to

$$
\begin{aligned}
& f=1+2 \sqrt{2} \sin P_{1}^{2} y e^{\eta_{1}}+e^{2 n_{1}}, \\
& \eta_{1}=P_{1} x-\frac{1}{2} P_{1}^{3} t
\end{aligned}
$$

which yields

$$
\begin{aligned}
& \mathrm{g}=\sqrt{2} \mathrm{P}_{1}^{2} \cos \mathrm{P}_{1}^{2} \mathrm{y} \mathrm{e}^{\mathrm{n}_{1}}, \\
& \mathrm{~h}=-\frac{\sqrt{2}}{2} \mathrm{P}_{1}^{4} \sin \mathrm{P}_{1}^{2} \mathrm{y} \mathrm{e}^{\mathrm{n}_{1}} .
\end{aligned}
$$


For $\mathrm{y}=0, \mathrm{~h}$ becomes zero and (35a) and (36a) give another soliton (cKdV, soliton) solution of (16),

$$
\begin{aligned}
& u=\frac{P^{2}}{2} \operatorname{sech}^{2}\left(P_{1} x-\frac{1}{2} P_{1}^{3} t\right), \\
& \phi=\frac{\sqrt{2}}{2} P_{1}^{2} \operatorname{sech}\left(P_{1} x-\frac{1}{2} P_{1}^{3} t\right) .
\end{aligned}
$$

It is noted that, if we take $a_{1}=a_{2}=1$ and $y=0$ in the above procedure, we obtain the one-soliton solution of a coupled equation,

$$
\begin{aligned}
& u_{t}=\frac{1}{4} u_{x x x}+3 u u_{x}+3 \omega_{x} \\
& \omega_{t}=-\frac{1}{2} \omega_{x x x}-3 u \omega_{x} .
\end{aligned}
$$

These procedure in obtaining solutions of (15) can be extended to the N-soliton state. From (24) we recover the KdV N-soliton solution by choosing $\xi_{j}(j=1,2, \cdots, N)$ as (28a). If we take $\xi_{j}$ as (28b) and $\xi_{N+j}$ as (28c) for $j=1,2, \ldots, N$, put $P_{j}=P_{j+N}$ and choose $a_{j}$ properly in the KP $2 \mathrm{~N}$-soliton solution, we obtain an N-soliton solution describing a multiple collision of the cKdV solitons. Moreover, it is also possible to get solutions showing interactions of the KdV solitons, (31), and the cKdV solitons, (37). For example, in the three-soliton solution of the KP equation, taking $\xi_{1}$ as (28a), $\xi_{2}$ as (28b) and $\xi_{3}$ as $(28 \mathrm{c})$, putting $\mathrm{P}_{2}=\mathrm{P}_{3}^{*}, \mathrm{a}_{2}=-\mathrm{a}_{3}=i$, and then choosing $\mathrm{y}=0$, we obtain a solution describing a head-on collision of the KdV and the ckdV solitons.

\section{References}

1) M.Sato and Y.Sato (Mori): RIMS Kokyuroku 388 (1980) 183; 414 (1981) 181 .

2) E.Date, M.Jimbo, M.Kashiwara and T.Miwa: RIMS preprint 394

3) R.Hirota and J.Satsuma: Phys. Lett. 85A (1981) 407. 